\title{
TAKING STEPS TO IMPROVE WORKING PRACTICE: A COMPANY EXPERIENCE OF METHOD TRANSFER
}

\author{
Björn Lundell ${ }^{1}$, Brian Lings ${ }^{2}$, Anders Mattsson ${ }^{3}$, and Ulf Ärlig $^{3}$ \\ ${ }^{1}$ Department of Computer Science, University of Skövde, Sweden; ${ }^{2}$ School of Engineering, \\ Computer Science and Mathematics, Department of Computer Science, University of \\ Exeter,UK; ${ }^{3}$ Combitech Systems AB, Jönköping, Sweden
}

\begin{abstract}
Methods are vital for systems development in companies, but their effective transfer into practice necessitates an understanding of the many factors affecting success. It can be extremely expensive to adopt a method in order to evaluate its suitability for a given context; what is required is a progressive commitment according to feedback. This paper documents the experience of progressive method transfer within one company.
\end{abstract}

Key words: method transfer, experience of transfer, working practice

\section{INTRODUCTION}

Effective use of methods is vital for competitive advantage in systems development contexts. The changing nature of technologies and requirements necessitates flexibility with respect to method adoption, and organisations need a systematic approach to the continuous improvement of work practices. In this paper we consider the transfer of a method into Combitech Systems $\mathrm{AB}^{1}$ (hereafter referred to as Combitech), an IT company within the SAAB group ${ }^{2}$. Combitech had become interested in the possible use of a method to support work practice improvement activities within the company.

\footnotetext{
${ }^{1}$ http://www.combitechsystems.com

${ }^{2}$ http://www.saab.se
} 
The method under transfer is the $2 G$ method, a qualitative method evolved for use in socio-technical domains. It was originally developed for evaluation of CASE-tools in specific usage contexts, but its scope of application has subsequently been broadened - a potential indicated in the method documentation (Lundell and Lings, 2003).

A number of studies of transfer of the $2 G$ method have previously been undertaken in a number of different company contexts (e.g. Lings and Lundell, 2004; Rehbinder et al., 2001). These have allowed us to theorise about effective processes of method transfer and influenced the approach taken to transfer at Combitech. In the rest of this paper we describe the progressive approach adopted, and reflect on the experience of transfer so far.

\section{ON THE METHOD BEING TRANSFERRED}

There is an underlying assumption behind the $2 G$ method that evaluation is a socio-technical activity, and that a key early activity in any evaluation is the development of a reference framework. Two inter-related frameworks are developed during an application of the $2 G$ method. To produce them, interview and other data is analysed both from an organisational and a technological perspective, using a two-phase process. In the first phase, the focus is on organisational need; in the second phase the focus shifts to how needs might be met through current technology (where technology is interpreted broadly, for example to include IS development methods). The $2 G$ method is not prescriptive, but gives clear guidelines on the use of different kinds of data source, and how frameworks may be evolved. It is important to the method that both phases in its application take place in the organisational setting in which the technology under investigation would be used. The method is intended as a general method, scalable according to context.

\section{ON METHOD DEVELOPMENT AND TRANSFER}

We can usefully consider seven steps in the development and transfer of a method, namely:

1. Method development and documentation

2. Method application by the method developer in a controlled context

3. Method application by the method developer in a company context

4. Method transfer to a method user within a controlled context 
5. Method transfer to a non-company method user within a company context

6. Method transfer to a company method user within a company context

7. Diffusion within a company already exposed to the method

From the point of view of transfer, the importance of the first three steps is to establish a clear conceptual framework for a method. This means that its underlying value systems must be transparent, and any assumptions about method use must be clear. It is important that they match those of any intended company context. The fourth step allows initial checks that the method is well enough established to be transferred to a user not previously exposed to it. This is a check that it can be learned and is usable at least in a controlled context. Both of these steps are necessary, but the real test of transfer comes only when a method is transferred into its intended context for usage. For example, the use of novices within a classroom setting may say little about an experienced practitioner's perception of a method within a company project.

The main concern of this paper is with steps 5 and 6 , where a method is transferred in to a real company context. Moody (2002) notes, from experiences of use of a data modelling method in a large commercial organisation, that "it is very difficult to get new approaches, especially those developed in academic environments, accepted and used in practice." (p. 393) Shanks (1996) ascribes this to the inherent risks and expenses for the organisation involved. For successful method transfer, therefore, such risks and expenses must be minimised.

The final, diffusion, step is beyond the scope of this paper but is the motivation behind Combitech's interest in the current transfer studies: the $2 G$ method is seen to have wider applicability throughout the company.

\section{THE COMBITECH EXPERIENCE}

The method receivers are consultants working in Combitech, and the specific context for initial $2 G$ method transfer is a development project undertaken within the company.

Combitech is a medium sized, geographically distributed enterprise working with advanced systems design and software development, electronic engineering, process optimisation and staff training. It has approximately 230 employees and covers a broad spectrum of business areas such as defence, aviation, vehicles, medical industry and telecom.

The company has a long experience of systematic method work and model driven systems development. In several development projects UML is used (e.g. Mattsson, 2002), but other methods and techniques are used as 
well. Development tools, such as Rational Rose (from Rational) and Rhapsody (from Ilogix) are also used in some projects.

From the company's perspective, $2 G$ was of interest because it offered a rich and systematic way of dealing with socio-technical phenomena. Managers had been made aware of earlier application of the $2 G$ method with respect to contextualised CASE tool evaluation, and the company had a general interest in tool-assisted model-driven development.

The process started with round table talks about previous applications of the method, and potential contexts within the company where it might be applicable. This led to the specification of a pilot project, in which a university researcher would be seconded to Combitech for a four month period to act as a method user (step 5 above). This was seen as an efficient way in which to gauge stakeholder reactions to the method. It also gave the company access to a continuous dialogue about the method and its potential usefulness within the company.

A consultant software engineer elected, with encouragement and full support from management, to follow the application in detail in order to be able to apply it internally. The dialogue established was seen as a positive and lightweight, hands-on introduction to the method. There was a strong sense of involvement by the software engineer, and the 4 month duration was seen to offer time for reflection and critical thinking throughout the process. However, during weeks with high short-term demands and other commitments, it was more difficult to preserve the momentum on this longer-term activity. It was felt that the extra load on stakeholders would be lightened if method application could be pursued on a more contingent basis.

It was important that the method could be viewed with appropriate scepticism, as it was being undertaken by an 'outsider' with no management commitment to eventual deployment. This placed it as a limited study, its role being as much for professional development as for method evaluation.

On completion of the pilot, Combitech were then in a good position not only to assess stakeholder reaction to the method, but also to judge its potential within the company. In particular, discussions ensued on how to apply the $2 G$ method in the company's ongoing work on method tailoring. From the company's perspective, there was a perceived competitive advantage offered by the $2 G$ method in supporting activities in method tailoring in a structured way. The pragmatic and strategic frameworks central to the $2 G$ method, interpreted as the how and why with respect to development methods, were seen as a means of contributing in two ways: firstly, for continuous improvement actions; secondly to assist in the initiation of new staff into company culture and working practices.

Having committed to deploying the method within the company, the next step (step 6 above) is to transfer the method fully to a company member - in 
this case the 'shadow' from the pilot project. The focus for this study is a tailoring of a development method, primarily influenced by RUP but also with other influences. At this stage of the process, mentoring is seen as the most effective mechanism to assist in method transfer. The mentor could be any method expert, but in this case is one of the method developers. This will be supplemented by stakeholder seminars, involving method experts and company members, for more reflective feedback and analysis.

A number of issues have been raised in the process of executing step 6. The major one concerned finding an appropriate project, which needed to be: representative of intended usage; internal; not too limited; and with reasonable planning time. It was perceived that there was no risk in applying the method, but it did demand stakeholder time - and this needed to be planned in the light of commitments. This is not straightforward for a consultancy company, with great variability in project sizes and lead times. The real need was to find a window for the intended method user just prior to the target project for analysis (in this case, method tailoring for a software development project). This window needed to be close to the project, large enough to evolve a useful framework, and at a time when interviews with relevant stakeholders could be scheduled. On the other hand, post-project analysis was felt to be less time-critical.

It is beyond the scope of the current project to consider step 7 in the process, which at the very least must await analysis of the experience of step 6. This is the stage at which costs could increase sharply, as other consultants are trained with the method. However, the cost would be ameliorated by the dissemination activities implied within step 6 , and management would already have had clear indications of the method's suitability and acceptability to stakeholders - i.e. risks would have been considerably reduced.

\section{SUMMARY}

We have identified seven steps in the process of method development and transfer, from the development of a method through to its full deployment within a company. From a company perspective, the most important of these are steps 5 through 7: from a pilot application of the method using an external 'consultant', through transfer to member of the company and finally diffusion within the company. We have taken as an example the transfer of the $2 G$ method into Combitech Systems AB, noting how the multiple steps can reduce risk and increase the likelihood of stakeholder acceptance. The overall experience for the company has been positive. In particular, it is felt that exchanges between consultants based on 
tacit knowledge have been increased and given greater structure. This has also facilitated reflection and created greater consensus within the group, leading to easier acceptance of change and therefore increased adaptability. This experience is consistent with methods as a means of improving current work practices rather than as prescriptive ways of working.

\section{ACKNOWLEDGEMENTS}

The authors are grateful to Hanna Zaxmy, the external method user for step 5 in the transfer process.

\section{REFERENCES}

Lings, B. and Lundell, B. "On transferring a method into a usage situation," in IFIP WG 8.2 IS Research Methods Conference: Relevant Theory and Informed Practice, 15-17 July, Manchester, 2004, Kluwer (accepted for publication).

Lundell, B., and Lings, B. "The $2 G$ method for doubly grounding evaluation frameworks," Information Systems Journal (13:4), 2003, pp. 375-398.

Mattsson, A. "Modellbaserad utveckling ger stora fördelar, men kräver mycket mer än bara verktyg," On Time, April, 2002 [www.ontime.nu] (in Swedish).

Moody, D.L. "Validation of a method for representing large entity relationship models: an action research study," in European Conference on Information Systems, 6-8 June, Gdansk, Poland, 2002, pp. 391-405.

Shanks, G. Building and Using Corporate Data Models, Ph.D. thesis, Monash University, Melbourne, 1996.

Rehbinder, A., Lings, B., Lundell, B. Burman, R. and Nilsson, A. "Observations from a Field Study on Developing a Framework for Pre-Usage Evaluation of CASE Tools," in New Directions in Information Systems Development: IFIP WG 8.22001 Conference, N.L. Russo, B. Fitzgerald, and J.I. DeGross (eds.), Kluwer, Boston, pp. 211-220. 